\title{
SISTEMA FECHADO DE ASPIRAÇÃO ENDOTRAQUEAL NA PREVENÇÃO DA HIPOXEMIA
}

Sonia Aurora Alves Grossi*

GROSSI, S.A.A. Sistema fechado de aspiração endotraqueal na prevenção da hipoxemia.

Rev.Eso.Enf.USP, v.29, n.1, p.26-33, abr. 1995.

Este trabalho apresenta uma revisão dos estudos relativos às indicações eficácia e efeitos indesejaueis decorrentes do uso do sistema fechado de aspiração endotraqueal. Apesar da dificuldade na generalização dos resultados dos trabalhos analisados, foi posslvel concluir que o uso do "trach-care" tem sua efetividade com: provada na manutençio da normoxia da maioria dos pacientes entubados e em ven. tilaçăo artificial.

UNITERMOS: Intubaçáo. Assistencia de Enfermagem. Anoxemia.

\section{INTRODUÇÃO}

A manutenção da permeabilidade das vias aéreas tem sido o maior desafio e o principal objetivo na assistência de enfermagem a pacientes entubados e em ventilação artificial. Dentre as medidas de enfermagem que asseguram a consecução deste objetivo a aspiração endotraqueal é considerada um procedimento necessário e rotineiro. Entretanto, não é isento de riscos, expondo os pacientes a sérias complicações, como: arritmias, laringoespasmos, traumatismos da mucosa traqueal, microatelectasias, hipoxemia, insaturação do oxigênio, bradicardias, hipertensão arterial, aumentos da pressão intracraniana, infecções, ansiedade, alterações na pressão parcial de gás carbônico, cianose, broncoconstrição e parada cardíaca.

Destas intercorrências, a hipoxemia tem sido apontada pela maioria dos investigadores como uma das mais sérias.

\footnotetext{
* Aluna da Pós-Graduaçáo da Escola de Enfermagem da USP e Professora Adjunta da Universidade do Sagrado Coraçáo.
} 
Segundo NIELSON ${ }^{13}$, a aspiração ocasiona uma irritação nas vias aéreas provocando estimulação vagal, com conseqüente broncoespasmo. Além disto, a excessiva pressão negativa reduz a oferta de oxigênio aos pulmões e gera microatelectasias. Como conseqüencia ao broncoespasmo e a atelectasia o paciente desenvolve hipoxemia que, associada à estimulação vagal, desencadeia graves bradicardias, com vaso constrição coronariana comprometendo seriamente o débito cardíaco e o fornecimento de sangue aos tecidos.

Hipoxemia é usualmente definida como um declínio significante na pressão parcial de oxigênio no sangue arterial $\left(\mathrm{PaO}_{2}\right)$, abaixo de $65 \mathrm{~mm} \mathrm{Hg}$ aproximadamente, associado a um rápido declínio na curva de dissociação de hemoglobina, neste ponto. Como a maioria dos pacientes entubados e em ventilação mecânica apresentam alterações nos níveis dos gases sangüíneos por doenças preexistentes, uma pressão parcial de oxigênio no sangue arterial $\left(\mathrm{PaO}_{2}\right)$ de $65 \mathrm{mmHg}$ ou uma saturação de oxigênio $\left(\mathrm{SaO}_{2}\right)$ em torno de $93 \%$ pode não ser parâmetro apropriado para determinar hipoxemia, individualmente. Por este motivo, é mais prudente definir hipoxemia como a queda da pressão parcial de oxigênio no sangue arterial $\left(\mathrm{PaO}_{2}\right)$, abaixo dos níveis basais do próprio paciente, antes do procedimento aspirativo ${ }^{15}$.

A constatação da ocorrência è da gravidade da hipoxemia ocasionada pelo procedimento aspirativo motivou muitos pesquisadores a desenvolver e avaliar vários métodos para garantir uma oferta de oxigênio adequada durante a aspiração endotraqueal. Um destes métodos consiste na utilização do sistema fechado de aspiração endotraqueal.

$\mathrm{O}$ conhecimento dos resultados de trabalhos que avaliaram os efeitos do emprego do sistema fechado na manutenção da normoxia pode auxiliar o enfermeiro a planejar o cuidado de seus pacientes. Assim, o objetivo deste trabalho é apresentar uma revisão de pesquisas sobre o assunto organizando as informações obtidas, ressaltando os aspectos concordantes, conflitantes e apontando as causas existentes sobre o tema proposto.

\section{DISCUSSÃO DA LITERATURA}

O sistema fechado de aspiração endotraqueal consiste na realização do procedimento aspirativo sem a desconexão do ventilador artificial do pacien. te. Para que isso fosse possível foi idealizado na década de 1970, um adaptador de cânula endotraqueal, com uma abertura lateral para permitir a entrada do cateter de aspiração. Desta forma os pacientes poderiam ser aspirados sem interrupção da ventilação e mantendo a pressão expiratória positiva final (PEEP) o que, teoricamente, reduziria a hipoxemia induzida pela sucção ${ }^{17}$.

Alguns estudos foram realizados para avaliar os efeitos deste adaptador 
sobre os niveis da pressão parcial de oxigênio no sangue arterial $\left(\mathrm{PaO}_{8}\right)$, saturação de oxigênio $\left(\mathrm{SaO}_{2}\right)^{1,8,10,10}$. BODAI' através de seu estudo com sete pacientes em falência respiratória (fração inspirada de oxigênio $\left(\mathrm{FiO}_{2}\right) \cdot 40$ a $100 \%$ ) e (pressão expiratória positiva final (PEEP) $\cdot 8 \cdot 20 \mathrm{cmH}_{2} \mathrm{O}$ ), comparou os efeitos do uso do adaptador associaido a suspiros, através do ventilador em condiçðes basais, antes e durante a aspiraçăo, com ventilaçōes através da bolsa de ressuscitação manual a $15 \mathrm{I} / \mathrm{min}$ de oxigênio nos mesmos minutos. Os resultados mostraram que apesar dos níveis da pressão parcial de oxigênio no sangue arterial $\left(\mathrm{PaO}_{2}\right)$ terem decrescido em ambos os procedimentos a queda média foi significativamente maior quando 0 adaptador năo foi utilizado. $O$ fato do autor não ter monitorizado a concentração de oxigênio $\theta 0$ volume de ar durante o uso da bolsa de ressuscitação manual compromete os resultados deste estudo, pois é possivel que a bolsa de ressuscitação manual não tenha deliberado quantidade similar de oxigênio, para permitir uma comparação válida.

A efetividade do uso do adaptador na prevenção da hipoxemia demons. trada em alguns estudos 3,4 não tem sido reconhecida ${ }^{1,10,28} \theta$ até contra-indicada, pois, segundo DOUGLAS; LARSON ${ }^{10}$, a tendência à recuperação da pressão parcial de oxigênio no sangue arterial $\left(\mathrm{PaO}_{8}\right)$ e saturação de oxigênio $\left(\mathrm{SaO}_{2}\right)$ aos níveis iniciais à aspiração foi significativamente mais evidente nos pacientes sem adaptador, mesmo quando tratados com altos niveis de pressão expiratoria positiva final (PEEP).

Segundo STONE; TURNER ${ }^{17}$ apesar de os pacientes não serem desconectados do ventilador durante a sucção traqueal, com o uso de adaptadores, um escape de ar ao redor do cateter favorece perdas de pressão inspiratória e expiratória. Outro inconveniente deste sistema é a criação de uma pressão negativa dentro das vias aéreas que pode resultar em atelectasias e colapso alveolar, pois, grandes pressões negativas podem ser geradas quando o fluxo de gás do ventilador for menor ou igual ao fluxo de aspiração.

Tendo em vista os resultados destes trabalhos, conclui-se que o desen. volvimento de um maior número de pesquisas com monitorização da pressão negativa desenvolvida nas vias aéreas e suas conseqüências deveriam ser realizadas para permitir uma avaliação mais adequada desta té-cnica.

Um novo dispositivo, para garantir uma oferta de oxigênio, adequada, durante a aspiracão endotraqueal, surgiu nos meados da década de 1980 . Trata-se de um sistema fechado de aspiração "trach-care", avaliado por diversos pesquisadores, nos últimos anos $2,3,11,12,14,16,19$. Consiste em um cateter de aspiração especial e de múltiplo usio, que permite a aspiração sem a interrupção da ventilação, podendo ser usado em todos os pacientes entubados e traqueostomizados, especialmente aqueles que necessitam de aspirações frequentes, manutenção da pressão expiratória positiva final (PEEP) e com infecção respiratória ${ }^{2} 12$.

O dispositivo "trach-care" é constituído de três componentes: o primei- 
ro componente é uma peça de forma de $\mathrm{T}$ com quatro aberturas, sendo que uma delas permite a conexão do dispositivo ao paciente através da cânula endotraqueal ou de traqueostomia, duas delas possibilitam a instalação do ventilador à direita ou esquerda do leito e a outra permite a entrada do cateter de aspiração; o segundo componente é o catéter de aspiração protegido por um saco de plástico e o terceiro componente é uma válvula de controle conectada no final do cateter de aspiração e que possibilita a ligação à rede de vácuo ${ }^{12}$.

As vantagens potenciais de um sistema fechado de sucção traqueal incluem a manutenção da oxigenação e da pressão expiratória positiva final (PEEP) durante o procedimento aspirativo, prevenção da contaminação cruzada entre os pacientes, prevenção da contaminação do trato respiratório por microrganismos ambientais, proteção da equipe e redução do custo com o procedimento, pois utiliza somente um cateter nas 24 horas e dispensa o uso de luvas ${ }^{2,12,14}$.

A manutenção da oxigenação sangüínea durante a aspiração endotraqueal tem sido uma das principais razões para a indicação do uso do sistema fechado de aspiração endotraqueal. Teoricamente, se a ventilação mecânica é mantida durante a sucção, a oxigenação e a pressão expiratória positiva final (PEEP) também poderiam ser mantidas e as complicações associadas à hipoxemia, reduzidas.

Os resultados dos estudos analisados mostraram os efeitos positivos da utilização do sistema fechado de aspiração sobre a pressão parcial de oxigênio no sangue arterial $\left(\mathrm{PaO}_{2}\right)^{5,8.20}$, saturação de oxigênio $\left(\mathrm{SaO}_{2}\right)^{14}$, saturação venosa de oxigênio $\left(\mathrm{SvO}_{2}\right)^{6}$, índice de transporte de oxigênio $\left(\mathrm{IDO}_{2}\right)^{11}$ e tempo de recuperação, após a sucção, em pacientes com e sem pressão expiratória positiva final (PEEP). Além disso, CARLON et al. ${ }^{5}$ demonstraram que os níveis da pressão parcial de oxigênio no sangue arterial $\left(\mathrm{PaO}_{2}\right)$ deterioraram de modo significativo, nos pacientes com pressão expiratória positiva final (PEEP) $>10 \mathrm{~cm}$ de $\mathrm{H}_{2} \mathrm{O}$, quando o sistema aberto convencional foi utilizado, o mesmo não acontecendo com o sistema fechado. Resultados semelhantes foram encontrados por CLARK et al. ${ }^{6}$ com relação às médias da saturação venosa de oxigênio $\left(\mathrm{SvO}_{2}\right)$ após a aspiração, em amostra de 189 pacientes, sendo que 125 destes faziam uso de pressão expiratória positiva final (PEEP).

Apesar de estes estudos terem comprovado a eficiência do sistema fechado de aspiração endotraqueal na preservação da oxigenação sangüínea, faz-se necessário ressaltar que variantes importantes como pressão, fluxo e duração da sucção, tempo e concentração e volume de oxigenação, quando mencionadas, foram bastante distintas.

CRAIG et al. ${ }^{7}$ avaliaram a necessidade de hiperoxigenação associada ao sistema fechado de aspiração e concluiram que ela não se faz necessária somente em pacientes em ventilação mandatória intermitente; entretanto é 
recomendada naqueles em ventilação assistida-controlada, pois a queda nos níveis de saturação de oxigênio $\left(\mathrm{SaO}_{2}\right)$ foi significativamente maior neste grupo de pacientes, quando não foi associada hiperoxigenação ao sistema fechado de aspiração. $O$ modo de ventilação, portanto, também afetou os resultados do estudo. Uma das razões possíveis, para explicar esta ocorrência no modo de ventilação assistida-controlada, é que ciclagens rápidas do venti. lador comumente ocorrem durante o procedimento aspirativo, podendo ocasionar remoções de oxigênio maiores do que a oferta ${ }^{14}$.

O modo de ventilação, também considerado uma variável importante ${ }^{14}$, não foi mencionado nos trabalhos analisados; entretanto, a maioria dos pesquisadores associou alguma forma de hiperoxigenação $0^{6,11,14}$ ou hiperoxigenação com hiperinflação ${ }^{5}$, antes e durante a sucção, evidentemente através do ven. tilador.

Os parâmetros cardiovasculares analisados em alguns estudos comparativos entre os sistemas aberto e fechado não evidenciaram alterações sig. nificativas na pressão arterial, freqüência cardíaca, indice cardíaco (IC) e indice sistólico (IS) entre os métodos ${ }^{6,8,11}$.

Uma suposta desvantagem potencial do sistema fechado está relacionada à possível multiplicação de microrganismos dentro e fora do cateter, quando o mesmo não está em uso e a conseqüente reinoculação destes microrganismos de volta à traquéia, a cada nova aspiração ${ }^{12}$. Apenas dois estudos publicados, enfocando estes aspectos microbiológicos, foram identifica$\operatorname{dos}^{911}$. Este risco de autocontaminação não foi evidenciado através do trabalho de RITZ et al. ${ }^{16}$, onde a análise estatística não mostrou diferenças significativas na freqüência e magnitude da contaminação entre os dois cateteres. DEPPE et al. ${ }^{9}$ através de um estudo prospectivo e randomizado com uma amostra de 85 pacientes, mostraram que o sistema fechado de aspiração aumentou significativamente a incidência de colonização, quando comparada com o sistema aberto; entretanto, não aumentou a incidência de pneumonia nosocomial. Foi demonstrada em uma análise que a probabilidade de sobrevida sem o desenvolvimento de pneumonia nosocomial foi significativamente maior entre os pacientes aspirados pelo sistema fechado. Com base nestes resultados e, objetivando reduzir a freqüência de infecção, é imperioso que a troca do cateter seja feita a cada 24 horas e que o sistema seja irrigado com solução salina após cada aspiração ${ }^{2}$.

Do mesmo modo como acontece com o uso de adaptadores, algumas evidências têm indicado que pressões negativas nas vias aéreas podem se desenvolver quando a sucção endotraqueal ocorre em sistema fechado. Essa pressão negativa poderia impedir a manutenção da pressão positiva inspiratória, o suprimento de oxigênio, a preservação da pressão expiratória positiva final (PEEP) e ainda causar atelectasias e hipoxemia. A principal causa para o surgimento desta pressão negativa, neste sistema, seria a incapacidade do ventilador em suprir rápida e adequadamente os gases aspira- 
dos através do cateter de suç̧ão ${ }^{19}$. TAGGART et al. ${ }^{19}$ com o objetivo de delinear as pressões das vias aéreas durante a aspiração através do sistema fechado e em múltiplas combinações de ventiladores e seus controles, manipularam as variáveis modo de ventilação, fluxo inspiratório, sensibilidade e pressão expiratória positiva final (PEEP), num estudo "ïn vitro". Os resultados evidenciaram que a pressão negativa manteve-se acima de $-10 \mathrm{~cm}$ de $\mathrm{H}_{2} \mathrm{O}$ sempre que fluxos inspiratórios, a partir de $25 \mathrm{I} / \mathrm{min}$, foram utilizados, exceto quando da utilização do respirador Puritan-Bennett 7200, onde foram necessários fluxos maiores que $25 \mathrm{I} / \mathrm{min}$ para impedir pressões negativas menores que $-10 \mathrm{~cm} \mathrm{H}_{2} \mathrm{O}$. No modo controlado de ventilação, com todos os fluxos inspiratórios testados, com ou sem prẹssão expiratória positiva final (PEEP), as pressões negativas geradas foram menores que $-50 \mathrm{cmH}_{2} \mathrm{O}$,o que permitiu aos autores concluir que o sistema fechado não deveria ser utilizado, neste modo, antes da reaplicação do estudo em seres humanos.

A aceitação do dispositivo "trach-care" ainda é controvertida na prática hospitalar levando em consideração algumas dificuldades na sua utilização e o custo ${ }^{14}$. Entretanto, o uso do cateter fechado tem-se mostrado bastante conveniente ao pessoal de enfermagem, pela prontidão e facilidade com que a aspiração pode ser realizada, reduzindo a ansiedade dos pacientes ${ }^{2,916}$.

\section{CONCLUSÕES}

Levando em consideração o estado atual de conhecimento neste tema, as observações pessoais e os aspectos concordantes da literatura compulsada como particularidade desta pesquisa, conclui-se que:

- o uso do "trach-care" é comprovadamente eficiente na manutenção da normoxia nos pacientes em ventilação mandatória intermitente, não sendo necessária nenhuma forma adicional de oxigenação;

- nos pacientes em ventilação assistida-controlada é recomendado a utilização de fluxos inspiratórios a partir de $25 \mathrm{I} / \mathrm{min}$ e associação de hiperoxigenações ao sistema fechado de aspiração;

- nos pacientes em ventilação controlada, o uso do "trach-care" deverá ser indicado com cautela até que estudos adicionais, com monitorização das pressões negativas geradas nas vias aéreas pelo uso do sistema fechado de aspiração, sejam realizados. 
(GROSSI, S.A.A. The closed tracheal system suctioning in prevention of the hypoxemia. Rev.Esc.Fnf.USP, v.29, n.1, p.26-33, Apr. 1995.

This paper shows a revision of the studies about indications efficacy and dangerous effects from the use of the closed tracheal system suctioning. In spite of the difficulties in the generalization of the results of the analysed works it was possible to conclude that the "trach-care" has its comproved efficiency in the maintenance of $\mathrm{PaO}_{2}$ in most intubated and ventilated patients.

UNITERMS: Intubation. Nursing care. Anoxemia.

\section{REFERENCIAS BIBLIOGRÁFICAS}

BAKER, P. et al. Endotracheal suctioning techniques in hypoxemic patients. Resp. Care, v. 28 , p. 1563-8. 1983 .

BIRDSALL, C. How do you use a closed suction adapter? Am. J. Nurs, v. 86, p. 1222-3, 1986.

BODAI, B.I. Ameans of suctioning without cardiopulmonary depression. Heart \& Lung, v. 11 , p. $172-6,1982$.

BODAI, B.I. et al. A clinical evaluation of an oxigen insuflation/suction catheter. Heart \& Lung, v. 16 , p. 39-46, 1987.

CARLON, G.C. et al. Evaluation of a closed tracheal suction system. Crit. Care Med., v. 15, p. $522-5,1987$.

CLARK, A.P. et al. Effects of endotracheal suctioning on mixed venous oxigen saturation on heart rate in critically ill adults. Heart \& Lung, v. 19, part 2, p. 552-7, 1990.

CRAIG, K.C. et al. Prevention of arterial oxygen desaturation during closed-airway endotracheal suction: effect of ventilator mode. Resp. Care, v. 29, p. 103-7, 1984.

DEMAJO, W. Effects os suctioning patients without interruption of ventilation. Toronto, s.d. Imimeografado/.

DEPPE, S.A. et al. Incidence of colonization, nosocomial pneumonia, and mortality in critically ill patients using a Trach Care suction system versus an open-suction system: prospective, randonized study. Crit. Care Med., v. 18, n. 12, p. 1389-93, 1990.

DOUGLAS, S.; LARSON, E.L. The effect of a positive end expiratory pressure adapter on oxigenation during endotracheal suctioning. Heart \& Lung, v. 14, n. 4, p. 396-400, 1985.

MATTAR, J.A. et al. A comparative study of oxigen transport between open and closed methods of tracheal suctioning. Intensive and Critical Care Digest, 1992./No prelo/

MAYHALL, C.G. The trach care closed tracheal suction system: a new medical device to permite tracheal suctioning without interruption of ventilory assistence. Infect Control Hosp. Epidemeol., v. 9, p. 125-6, 1988. 
NIELSON, L. Potential problems of mechanical ventilation. Am. J. Nurs., v. 80, p. 220613,1980 .

NOLL, M.L. et al. Closed tracheal suction systems: effectiveness and nursing implications. AACN Clin. Issues Crit. Care Nurs., v. 1, p. 318-28, 1990.

RIEGEL, B.; FORSHEE, T. A review and critique of the literature on preoxygenation for endotracheal suctioning. Heart \& Lung, v. 14, p. 507-17, 1985.

RITZ, R. et al. Contamination of a multiple use suction catheter in a closed circuit system compared to contamination of a disposable, single use suction catheter. Resp. Care, v. 31 , p. $1086-89,1986$.

STONE, K.S.; TURNER, B. Endotracheal suctioning. Annu. Rev. Nurs. Res., v. 7, p. 27-49, 1989.

STONGE, D. et al. The effect of maintaining and removing positive end expiratory pressure during endotracheal suctioning. In: NATIONAL TEACHING INSTITUTE, 9, Newport Beach, 1982. Proceedings, Newport Beach, American Association of Critical Care Nurses, 1982 , p. 307.

TAGGART, J.A. et al. Airway pressures during closed system suctioning. Heart \& Lung, v. 17, p. $536-42,1988$.

WILHELM, D. et al. Prevention of endotraqueal suction indured arterial desaturation with a close airway system catheter. Minnesota, s.d. /Mimeografado/ 